\title{
Paridad y cuotas electorales en Europa*
}

\author{
Parity and electoral quotas in Europe \\ Paridade e cotas eleitorais na Europa
}

\author{
Cristiane Aquino de Souza*
}

\section{Resumen}

Este trabajo realiza un análisis del proceso de adopción de cuotas electorales legales de género en algunos países de Europa. El objetivo de la investigación ha sido comprender el contexto en el cual se han desarrollado las modificaciones legales, constitucionales o jurisprudenciales que han favorecido la adopción y aplicación de las cuotas y de la paridad. Por otro lado, se ha analizado la eficacia de estas medidas en los países europeos estudiados.

Palabras clave: Cuotas electorales. Paridad. Género. Europa.

\section{Abstract}

This work analyzes the adoption of electoral gender quotas in some European countries. The study intends to understand the development of legal, constitutional and jurisprudential modifications that have influenced the adoption of quotas and parity. In addition, it analyzes the efficiency of these measures in the European countries that have been studied.

Keywords: Electoral quotas. Parity. Gender. Europe.

* Este trabalho faz parte do projeto de investigação "Democracia e Paridade de gênero", financiado pelo Conselho Nacional de Desenvolvimento Científico e Tecnológico (CNPq) e pela Fundação Cearense de Apoio ao Desenvolvimento Científico e Tecnológico (FUNCAP).

* Doutora em Direito pela Universidade Autônoma de Madri (2011), mestra em Direito pela Universidade Federal do Ceará (2005), graduada em Direito pela Universidade Federal do Ceará (2002). Atualmente é professora adjunta da Universidade de Fortaleza. Fortaleza - Ceará Brasil. Email: casfortaleza@hotmail.com 


\section{Resumo}

Este trabalho analisa o processo de adoção, mediante lei, de cotas eleitorais de gênero em alguns países da Europa. O objetivo da investigação foi compreender o contexto no qual se desenvolveram as modificações legais, constitucionais ou jurisprudenciais que favoreceram a adoção e aplicação das cotas e da paridade. Por outro lado, analisou-se a eficácia destas medidas nos países estudados.

Palavras-chave: Cotas eleitorais. Paridade. Gênero. Europa.

\section{Introducción}

En Europa, las cuotas electorales legales de género han sido establecidas por diversos países en las décadas de 1990 y $2000^{1}$. En todos los casos, se ha previsto una cuota para cada sexo o para el sexo infrarrepresentado en el ámbito de las candidaturas de los partidos. En este trabajo, se realiza un análisis del proceso de adopción de cuotas electorales legales en los siguientes países: Bélgica, Francia, Italia, Portugal y España. El objetivo de la investigación ha sido comprender el contexto en el cual se han desarrollado las modificaciones legales, constitucionales o jurisprudenciales que han favorecido la adopción y aplicación de las cuotas y de la paridad. Por otro lado, se ha analizado la eficacia de estas medidas en los países europeos estudiados.

\section{Bélgica}

Bélgica fue el primer país europeo que estableció tal tipo de cuotas $^{2}$. La Ley Smet-Tobback, de 24 de mayo de 1994, estableció que

\footnotetext{
En Europa, las cuotas electorales legales han sido adoptadas en Albania, Bélgica, BosniaHerzegovina, Eslovenia, España, Francia, Grecia, Irlanda, Italia, Macedonia, Polonia, Portugal y Serbia. International Institute for Democracy And Electoral Assistance (IDEA), en cooperación con la Universidad de Estocolmo. Disponible en http://www.quotaproject.org/about.cfm. [consultado 3/05/2012].

2 Sobre la experiencia de Bélgica, véase, entre otros, MEIER (1999) e MATEO DÍAZ (2003).
} 
las listas deben incluir un máximo de $75 \%$ de candidatos del mismo sexo (es decir, un mínimo de $25 \%$ ) para las elecciones locales y provinciales de 1994. La Ley previó un aumento de la cuota para un mínimo del 33\% a partir de las elecciones de 1999, año en el cual la reserva mínima se aplicó por primera vez en el ámbito de las elecciones generales. El número de diputadas elegidas pasó del $12 \%$, en 1995 , al $23 \%$ en 1999. No obstante, la mudanza más destacable ocurrió tras la puesta en marcha de una reforma constitucional y de otras alteraciones legislativas.

En febrero de 2002, se añadió un párrafo al artículo 10 de la Constitución, que garantiza la igualdad entre mujeres y hombres. Por otro lado, se insertó el artículo 11 bis, el cual establecía que la ley debe garantizar la igualdad entre hombres y mujeres en el acceso a los mandatos electivos y públicos. Este artículo también indicaba que los dos sexos deben estar representados en todos los órganos ejecutivos.

En el plano legal, en julio del mismo año 2002, se presentó un proyecto de ley que pretendía aprobar dos mecanismos: por un lado, la diferencia entre el número de candidatos(as) hombres y mujeres no debería ser superior a uno, es decir, se debería respetar la proporción del $50 \%$ de los puestos para cada sexo; por otro lado, las dos primeras posiciones de cada lista electoral deberían estar ocupadas por un hombre y una mujer. Los miembros de la Cámara de los representantes favorables a estas medidas intentaron, sin logro, aprobar el principio de alternancia hombre-mujer para toda la lista, lo que ellos denominan "principe de la tirette". La ley, aprobada en julio de $2002^{3}$, establece una disposición transitoria para ser aplicada en las elecciones de 2003: una mujer y un hombre deben estar presentes en las tres primeras posiciones de las listas. Se dispone que, en las elecciones posteriores, se deba aplicar esta regla para las dos primeras posiciones de las

Ley Especial de 18 de julio de 2002, de garantía de igual representación de hombres y mujeres en las listas electorales al Consejo de la Región Valona, al Consejo Flamenco y al Consejo de la Región de Bruselas-Capital. Por otro lado, también se puede destacar la aprobación de la Ley de 17 de junio de 2002, de garantía de igual representación de hombres y mujeres en las listas electorales al Parlamento Europeo. 
candidaturas. Se aprueba también la cuota del $50 \%$ y se prevé que su incumplimiento ocasiona el rechazo de la lista por las autoridades (MEIER, 2008). Aunque no se hubiera aprobado la alternancia entre los sexos en toda la lista, la norma transitoria que ha garantizado la presencia de un hombre y de una mujer en los tres primeros puestos de las listas parece haber ocasionado resultados eficaces (MATEO DÍAS, 2003), pues el porcentaje de mujeres en la Cámara de Representantes ascendió del $23,3 \%$ en 1999 , al $35 \%$ en 2003 ; en las elecciones de 2007, ha sido del 36,67\%, y en las de 2010, del 39,33\%. En el Senado, el ascenso ha sido del $28 \%$ en 1999 al $30 \%$ en 2003, un $32 \%$ en 2007 y un $42,5 \%$ en $2010^{4}$. En las diferentes legislaturas regionales, entre 1999 y 2004 , el porcentaje de candidatas elegidas aumentó del $11 \%$ al $19 \%$ en el parlamento valón, del $20 \%$ al $31 \%$ en el flamenco, y del $35 \%$ al $46 \%$ en el de la región de Bruselas-Capital, mientras que se mantuvo en el $24 \%$ en el de la comunidad germanófoba (MEIER, 2008).

En Bélgica, se adopta el sistema de listas cerradas y no bloqueadas (de voto preferencial múltiple opcional). Sin embargo, los votos preferenciales se aplican únicamente para el $50 \%$ de los escaños asignados, ya que el otro $50 \%$ se distribuye de acuerdo con el voto de lista. Así, la ubicación del candidato interviene en sus posibilidades de ser elegido toda vez que los ocupantes de las posiciones más altas se benefician de los votos de lista (MEIER, 2008). Por esta razón, se caracteriza la importancia de la exigencia de mandato de posición en la legislación sobre cuotas en el país, aunque el impacto de esta disposición esté limitado por el hecho de que la mitad de los escaños sean asignados en función de los votos preferenciales.

Es siempre difícil medir exactamente la influencia que las cuotas electorales ejercieron para el aumento del número de mujeres elegidas al Parlamento, ya que siempre existen otras variables, y en el caso de Bélgica, se puede citar, por ejemplo, la ampliación del tamaño de las circunscripciones (MEIER, 2008). No obstante, los números indican que

4 UNIÓN INTERPARLAMENTARIA. http://www.ipu.org/parline-e/parlinesearch.asp. 
estas medidas tuvieron éxito y contribuyeron para la ampliación de la proporción de parlamentares mujeres. Algunos factores que pueden ser destacados como positivos para eso son el alto porcentaje de la cuota $(50 \%)$, la exigencia de un sistema de alternancia entre los sexos en los dos primeros lugares de las listas y la sanción por incumplimiento consistente en el rechazo de la lista electoral. El sistema de listas cerradas y no bloqueadas con voto preferencial múltiple opcional aplicable para $50 \%$ de los escaños asignados al partido puede también haber contribuido, aunque en este caso dependa también de factores como la tendencia o no de los electores en optar por el voto preferencial y el atractivo electoral de las mujeres en el país.

\section{Francia}

En Francia, se ha intentado introducir una ley que estableciera cuotas electorales por razón de sexo desde 1979. En enero de ese año, la Ministra de la Familia y de la Condición Femenina, Monique Pelletier, propuso que se estableciera una reserva de un mínimo de $20 \%$ de los puestos de las candidaturas para las mujeres en el ámbito de las elecciones municipales de ciudades con más de 2.500 habitantes. En 1980, el primer ministro francés sometió al Parlamento un proyecto de ley que acogía la mencionada propuesta, pero con la adopción de la fórmula neutra, que se refería a la reserva para cada sexo y no expresamente para las mujeres. Aunque el proyecto hubiera sido aprobado por la Asamblea Nacional, no llegó a ser examinado por el Senado, debido a la clausura del período de sesiones por la convocatoria electoral.

Tras las elecciones de 1982, el ministro del Interior preparó un proyecto de ley de reforma de las elecciones municipales que, inicialmente, no contemplaba las cuotas. No obstante, la diputada socialista Gisèle Halimi propuso una enmienda que pretendía establecer una cuota del $30 \%$, porcentaje que fue rebajado para el $25 \%$, mediante modificación gubernamental. Este proyecto de ley fue aprobado por la Asamblea Nacional, pero fue impugnado ante el Consejo Constitucional por los diputados de la oposición que, no obstante, no cuestionaron la 
disposición que establecía las cuotas, sino otras disposiciones de la ley. Es destacable el hecho de que el Consejo Constitucional planteó de oficio la inconstitucionalidad de las cuotas, invalidando tal disposición (Decisión n.82-146 DC, de 18 de noviembre de 1982). En razón de este fallo, cesaron las propuestas de cuotas por un período (LENOIR, 2003).

En la década de los 1990, creció en Francia una movilización a favor de la paridad, es decir, de un número igual o aproximadamente igual de mujeres y hombres en las instituciones. Las elecciones legislativas de 1993 demostraron que no se había conseguido disminuir el carácter preponderantemente masculino del Parlamento (el porcentaje de diputadas era del $13,2 \%$ ), situación descrita como vergonzosa, ya que situaba al país en los últimos lugares de los Estados occidentales en términos de representación política femenina. Se solía comparar Francia con otros países y, en este contexto, la periodista Christine Leclerc, por ejemplo, escribió que "pese a su machismo y su condición de democracia emergente, España tiene tres veces más mujeres parlamentarias" (Le Monde, 19 de febrero de 1993) (JANSON, 2001). En 1993, se publicó en el periódico Le Monde el "Manifiesto de las 577 personas a favor de la paridad", cuyo grupo de signatarios estaba formado por 289 mujeres y 288 hombres pertenecientes a diversos ámbitos políticos. En 1995, el primer ministro francés, Juppé, fundó el Observatorio de la Paridad como órgano de investigación y consulta. En 1996, diez ex ministras publicaron el "Manifiesto de las diez en pro de la paridad" ( $L$ 'Express, 6 de junio de 1996), en el que se proponía, entre otras medidas, la institución de cuotas con el propósito de alcanzar el mínimo de un tercio de mujeres en las Asambleas y en el que se hacía una declaración favorable a la alteración de la Constitución, si fuera necesaria, para la introducción de las medidas requeridas. En 1997, había en torno a setenta y dos asociaciones en Francia trabajando a favor de la mayor presencia de las mujeres en el ámbito político.

Pese a la situación descrita, el Consejo Constitucional francés se basó en la anterior decisión de 1982 y declaró inconstitucional una disposición de un proyecto de ley que aseguraba la composición numérica igualitaria de hombres y mujeres en las listas para las elecciones 
de los consejeros regionales y de los consejeros de la Asamblea de Córcega (Decisión n. 98-407 DC, de 14 de enero de 1999). En época contemporánea a esta decisión, se intentó el cambio de situación a través de una reforma constitucional. El primer ministro francés, Lionel Jospin (de un partido de izquierda), consiguió, no sin reluctancia, el acuerdo del presidente francés, Jaques Chirac (de un partido de derecha), con el objetivo de proponer una enmienda a la Constitución para garantizar la paridad. La reforma constitucional, aprobada en marzo de 1999, añade un nuevo apartado en el artículo 3 de la Constitución, relativo a la soberanía, en el que se dispone que "la ley favorece el igual acceso de las mujeres y los hombres a los mandatos electorales y las funciones electivas". Se introduce también un nuevo apartado en el artículo 4, sobre los partidos políticos, que establece que ellos "contribuyen a la aplicación del principio enunciado en el último apartado del artículo 3 en las condiciones determinadas por la ley". Estas modificaciones pasaron a constituir la Ley Constitucional 99-59, de 8 de julio.

Tras la mencionada reforma constitucional, el parlamento francés aprobó la Ley 2000-493, de 6 de junio, tendente a favorecer el igual acceso a mujeres y hombres a los mandatos electorales y funciones electivas. Esta norma establece una paridad entre hombres y mujeres del $50 \%$ en las listas electorales para las elecciones municipales (en los municipios con 3.500 habitantes o más), senatoriales (para la mitad del Senado, pues sólo se aplica en los departamentos que eligen senadores por el sistema proporcional), regionales y europeas.

La lista debe ser invalidada en caso de inobservancia de la comentada norma. Por otro lado, se establece la estricta alternancia de mujeres y hombres en toda la lista. En un primer momento, la Ley previa una estricta alternancia para el sistema de una sola ronda y una alternancia por bloques de seis candidatos cuando se trataba del sistema de dos rondas. Posteriormente, por medio de las reformas de 2003 y $2007^{5}$, se exige la alternancia estricta para todas las elecciones

La Ley de 11 de abril de 2003 prevé la estricta alternancia para las listas regionales. La Ley de 31 de enero de 2007 introduce varias mudanzas: para las próximas elecciones legislativas, aumenta 
proporcionales. En las elecciones para los miembros de la Asamblea Nacional, que se celebran en consonancia con un sistema mayoritario de distritos uninominales, la no observancia de la paridad implica sanciones económicas.

La Ley 2000-493, de 6 de junio, fue sometida a un recurso ante el Consejo Constitucional francés, que, finalmente, dadas las alteraciones constitucionales, consideró legítimas las medidas adoptadas por la legislación, con excepción de aspectos puntuales (Decisión n. 2000-429 DC, de 30 de mayo de 2000) (MARTÍNEZ ALARCÓN, 2007).

La ley de paridad francesa ha tenido impacto positivo en ciertas elecciones. En los comicios municipales, aunque la aplicación de la paridad esté restringida a los municipios con 3.500 habitantes o más (que representan sólo el $7,7 \%$ ), se ha observado un incremento significativo de mujeres elegidas, que pasó del 27,5\% en 1998 al 47,6\% en 2004. En el caso del Parlamento Europeo, el porcentaje de mujeres en 1999 ya era alto $(40,2 \%)$ y creció al $43,6 \%$ en 2004 . Para los Consejos Regionales, hubo un crecimiento notorio, de $27,5 \%$ en 1998 al $47,6 \%$ en 2004 (SINEAU, 2008).

Sin embargo, en las elecciones a las asambleas departamentales, al Senado y a la Asamblea Nacional, los números de mujeres elegidas permanecieron bajos. En el caso de las asambleas departamentales, la justificación es sencilla, pues la Ley no obliga a la paridad, lo que caracteriza uno de sus puntos débiles. En relación con el Senado, la Ley sólo se aplica para elegir la mitad de los escaños senatoriales (en los distritos con representación proporcional). Ésta es una posible razón por la que sólo se pudo observar una limitada penetración femenina,

las sanciones económicas para los partidos que no presenten el $50 \%$ de candidaturas de cada sexo, con la posibilidad de un error del $2 \%$. Tales sanciones consisten en el $75 \%$ de la diferencia entre candidatos masculinos y femeninos. La Ley posibilita sustituciones para las asambleas departamentales y establece que suplente y suplido deben ser de sexos opuestos. La Ley pasa a exigir la alternancia estricta para las elecciones municipales con poblaciones de 3.500 habitantes o más. Por fin, la Ley prevé la paridad para los comités ejecutivos de las regiones y de los municipios de 3.500 habitantes o más.

6 UNIÓN INTERPARLAMENTARIA. http://www.ipu.org/parline-e/parlinesearch.asp. 
mediante el incremento de $5,9 \%$ en 1998 al $10,9 \%$ en $2001,16,9 \%$ en 2004 y un $21,9 \%$ en $2008^{6}$.

En las elecciones a la Asamblea Nacional, la Ley tampoco ha logrado aumentar el número de diputadas elegidas de forma considerable. Aunque el porcentaje de candidatas haya aumentado de menos de $25 \%$ en 1997 hasta el $38,9 \%$ en 2002 y un $41,6 \%$ en 2007 , la proporción de mujeres elegidas pasó del $10,9 \%$ en 1997 al 12,3\% en 2002 y un 18,9\% en 2007. Esto sucede, entre otras razones, porque, para las elecciones a la Asamblea Nacional, se aplica el sistema mayoritario de distritos uninominales, el cual perjudica la eficacia de estas medidas. Por otro lado, los grandes partidos, por tener buenas condiciones financieras, prefieren pagar las multas recibidas en lugar de cumplir la paridad. En 2002, la sanción anual ocasionada por este incumplimiento ha sido de más de 4 millones de euros para la UMP, 1,3 millones de euros para el Partido Socialista, 582.000 euros para la Unión para la Democracia Francesa y 119.000 para el Partido Comunista (SINEAU, 2008).

Otro factor destacable es que los partidos tienden a presentar más mujeres en los distritos electorales donde tenían menos probabilidades de ganar, ya que los datos indican que la influencia de un partido en un distrito electoral es inversamente proporcional al porcentaje de candidatas femeninas. Por eso, en 2007, aunque el $26,6 \%$ de los candidatos de la UMP eran mujeres, sólo el 14,3\% resultaron elegidas; y para el Partido Socialista, los porcentajes fueron del 46,5 y del $25,9 \%$ (SINEAU, 2008).

En razón de las mencionadas circunstancias, la ley de paridad francesa no ha tenido éxito en el sentido de aumentar de forma significativa el número de mujeres elegidas para la Asamblea Nacional y para el Senado.

\section{Italia}

Al igual que en Francia, en Italia, los intentos de imposición de cuotas electorales por medio de ley en la década de 1990 vieron 
denegada su constitucionalidad por parte de la Corte Constituzionale. En este período, fueron aprobadas varias normas que preveían reservas electorales por razón de sexo. La Ley n. 81/1993, de 25 de marzo de 1993, estableció un sistema de cuotas en las elecciones para el Consejo Municipal a fin de asegurar la presencia de al menos un tercio $(33 \%)$ de candidatos del mismo sexo en las listas electorales ${ }^{7}$. La Ley 227 , de 4 de agosto de 1993, relativa a la elección a la Cámara de los Diputados, dispuso que las listas presentadas en el ámbito regional para la atribución del $25 \%$ de los escaños con sistema proporcional deberían presentar mujeres y hombres en alternancia. La Ley n. 43, de 28 de febrero de 1995, referida a la elección de los consejeros de las regiones, estableció la presencia de un mínimo de $33 \%$ de candidatos del mismo sexo en las listas. Por otra parte, había normas análogas aprobadas en las leyes de algunas regiones con estatuto especial ${ }^{8}$.

El tema de las cuotas fue elevado a la Corte Constituzionale cuando se planteó una cuestión de constitucionalidad del artículo 5.2 de la Ley n. 81/1993, que establecía cuotas de un tercio para candidatos del mismo sexo en las listas de candidaturas a la elección del alcalde y del consejo municipal en las ciudades con población de hasta 15.000 habitantes. La Corte consideró esta norma inconstitucional por medio de la Sentencia n. 422, de 12 de septiembre de 1995, y extendió la declaración de inconstitucionalidad a todas las demás disposiciones legales mencionadas que establecían cuotas por razón de sexo en las listas de candidaturas.

Posteriormente, en la década de 2000, se aprobaron en Italia algunas reformas constitucionales que pretendían posibilitar que futuras medidas legales sobre cuotas electorales en razón de sexo se consideraran legítimas por parte de la Corte Constituzionale. La Ley

Artículos 5.2 y 7.1 de la Ley 81/1993: "En las listas de los candidatos, ninguno de los sexos podrá estar por norma representado en medida superior a dos tercios". Parte de la jurisprudencia ha comprendido que la expresión por norma (di norma) confería un carácter programático a la medida. Por esto, el legislador ha suprimido esta locución a través de la Ley 415/1993.

8 Ley n.3/1994 del Trentino-Alto Adigio, Ley n.14/1995 de Friuli-Venezia Giulia y Ley n.4/1995 de Valle de Aosta. 
Constitucional n. 2, de 31 de enero de 2001, relativa a la elección de los presidentes de las regiones con estatutos especiales ${ }^{9}$ y de la provincia autónoma de Trento y de Bolzano, estableció que "a fin de conseguir el equilibrio de la representación de los sexos, la ley promoverá condiciones de paridad para el acceso a las consultas electorales"10. Por otra parte, la Ley Constitucional n.3, de 18 de octubre de 2001, alteró el artículo 117 de la Constitución italiana para establecer, entre otras disposiciones, que "las leyes regionales removerán cualquier obstáculo que impida la plena paridad de hombres y mujeres en la vida social, cultural y económica y promoverán la paridad de acceso entre mujeres y hombres a los cargos electivos".

En razón de estas normas, algunas regiones aprobaron leyes para favorecer el equilibrio en la representación de los sexos. En la norma que regula la elección del Consejo Regional del Valle de Aosta, por ejemplo, se insertaron dos disposiciones. Una de ellas dispone que "la Región debe promover el equilibrio de la representación de los sexos y las condiciones de paridad para el acceso a las consultas electorales". La otra establece que "cada lista a la elección del Consejo Regional debe prever la presencia de candidatos de ambos sexos"11.

Pese a la "timidez" de esta norma, que se sitúa lejos de establecer un equilibrio entre los sexos en las candidaturas, su contenido fue impugnado ante la Corte Constituzionale por el Gobierno en septiembre de 2002. En este caso, la Corte italiana ha declarado infundada la cuestión de legitimidad constitucional planteada (Sentencia n. 49, de febrero de 2003). En esta sentencia, el Alto Tribunal italiano ha modificado, de forma sustancial, sus criterios interpretativos y ha afirmado que la normativa impugnada debería ser evaluada a la luz de un cuadro constitucional

Sicilia, Valle de Aosta, Cerdeña, Trentino-Alto Adige y Friuli-Venezia Giulia.

10 Esta norma queda insertada en cada uno de los estatutos especiales regionales, de conformidad con lo que dispone la Ley constitucional n. 2, de 31 de enero de 2001.

11 Se trata del artículo 3-bis, párrafos 1 y 2, respectivamente, de la Ley Regional n. 3, de 12 de enero de 1993, insertados por Ley Regional n. 21, de 13 de noviembre de 2002 (aprobada por el Consejo Regional el 25 de julio de 2002). 
que ha evolucionado respecto al que estaba en vigor en la época de la anterior decisión de 1995 (FJ4).

Hay que resaltar que, mientras esta cuestión de constitucionalidad estaba pendiente de decisión, estaba en proceso de deliberación un proyecto de ley de reforma del artículo 51.1 de la Constitución italiana, que fue aprobado en mayo de 2003, tres meses después de dictarse la referida sentencia. El artículo 51.1 ya establecía, y todavía establece, que "todos los ciudadanos de uno y otro sexo podrán acceder a los cargos públicos y puestos electivos en condiciones de igualdad, según los requisitos establecidos por la ley". Con la reforma, se ha añadido lo siguiente: "A tal fin, la República promueve, con apropiadas disposiciones, la igualdad de oportunidades entre mujeres y hombres"12. Teniendo en cuenta la importancia conferida por la doctrina y la jurisprudencia italianas al artículo 51 como criterio interpretativo en materia de igualdad, esta modificación, o mejor, esta adición a la Constitución presenta una significativa transcendencia para legitimar las cuotas electorales legales por razón de sexo.

En efecto, después de las mencionadas mudanzas constitucionales, entre las cuales se destaca la alteración del art. 51, varias regiones italianas han introducido cuotas electorales de género. Se pueden citar como ejemplos el art. 3.3 de la Ley Regional n. 2, de 2005, de Puglia; el art. 3.2 de la Ley Regional n. 2, de 2005, de Lacio; el art. 8.4 de la Ley Regional n. 25, de 2004, de Toscana; y el art. 10.2 de la Ley Regional n. 4, de 2009, de Campania. Estas disposiciones prevén que, en la lista electoral, ninguno de los dos sexos pueden estar representados en medida superior a dos tercios $(66 \%)$ de los candidatos. La Ley Regional n. 7, de 2005, de Sicilia, establece que, después del cabeza de lista, todos los candidatos deben seguir un orden de acuerdo con un criterio de alternancia entre hombres y mujeres (art. 2.8).

Esta reforma se estableció por medio del artículo 1 de la Ley Constitucional n. 1, de 30 de mayo de 2003. 
En lo que respecta la previsión de sanciones por incumplimiento de las cuotas, algunas leyes, como la de Puglia y la de Lacio, disponen que, si el partido viola estas reglas, deberá devolver los gastos electorales reembolsados, hasta un máximo de la mitad, en una medida directamente proporcional al número de candidatos excedentes en relación con el mínimo permitido para cada sexo. La Ley de Campania, por otra parte, impone la no admisión de la lista que no respeta el porcentaje establecido (art. 10.3).

La mencionada legislación de Campania (Ley Regional n. 4, de 2009) tiene una disposición sobre el sistema electoral que establece que, en la votación, el elector puede exprimir uno o dos votos de preferencia y que, en el caso de optar por emitir dos votos preferenciales, uno debe ser destinado a un candidato del género masculino y el otro a una candidata del género femenino de la misma lista, so pena de anulación de la segunda preferencia (art. 4.3). Este artículo ha tenido su legitimidad cuestionada ante la Corte Constituzionale, que, en la sentencia n. 4, de 14 de enero de 2010, ha considerado infundada esta cuestión de constitucionalidad. Como argumento, el Tribunal ha subrayado que la finalidad de la regla es alcanzar el equilibrio de la representación política de los dos sexos, en conformidad con lo que dispone el art. 51.1 de la Constitución y el art. 5.3 del Nuevo Estatuto de la Región de Campania. Además, considera que la norma no viola la libertad electoral porque la expresión de la doble preferencia es facultativa para el elector, que puede emitir solamente una destinada a un candidato de cualquiera de los dos sexos y añade que la regla no pre configura un resultado electoral ni altera de forma artificial la composición de la representación (FJ 3.3).

De conformidad con lo observado anteriormente, las cuotas electorales de género parecen estar consolidadas en varias regiones de Italia, incluso con disposiciones que incluyen otras medidas, como la que se acaba de mencionar. Sin embargo, y aunque exista un cuadro constitucional favorable, todavía no se han adoptado cuotas a nivel estatal, pese a haber existido intentos en este sentido (CECCHERINI, 2006). 


\section{Portugal}

En Portugal, la revisión constitucional de 1997 añadió a la Constitución del país dos normas relevantes para el tema de las cuotas por razón de sexo. Una de estas disposiciones establece que constituye una de las tareas fundamentales del Estado "[...] promover la igualdad entre hombres y mujeres" (párrafo ' $h$ ' del artículo 9). La otra norma dispone que

La participación directa y activa de hombres y mujeres en la vida política constituye condición e instrumento fundamental en la consolidación del sistema democrático, debiendo la ley promover la igualdad en el ejercicio de los derechos cívicos y políticos y la no discriminación en función del sexo en el acceso a los cargos públicos (artículo 109) (AMARAL, 2000, p. 172).

Tras estas modificaciones, en el año de 1999, el Partido Socialista presentó dos proyectos de ley que establecían cuotas por razón de sexo en las listas electorales. No obstante, tales propuestas no obtuvieron la aprobación del Parlamento.

En abril de 2006, la Asamblea de la República portuguesa aprobó una ley que establece una cuota mínima del 33,3\% para cada sexo en las listas de candidatura a la Asamblea de la Republica, al Parlamento Europeo y a las autarquías locales. Esta ley fue vetada por el presidente de la República, Cavaco Silva, por entender que la posibilidad prevista por la norma de no admisión de las listas no conformes con la mencionada disposición constituía una sanción excesiva y desproporcionada. En razón de esto, se alteró el texto de la normativa para establecer como sanción al partido incumplidor la reducción del montante de subvenciones públicas para las campañas electorales ${ }^{13}$. Tras la aprobación de esta

13 De conformidad con el art. 7.1 de la Ley Orgánica 3/2006, la subvención pública es reducida en el montante del $50 \%$ si uno de los sexos estuviera representando en porcentaje inferior al $20 \%$, o en el montante del $25 \%$ si la representación de uno de los sexos fuera superior al $20 \%$ e inferior al $33,3 \%$. 
segunda versión en el Parlamento, en julio, fue promulgada la Ley Orgánica 3/2006, conocida en el país como Lei da Paridade ${ }^{14}$.

Esta Ley obliga a la alternancia entre mujeres y hombres en las listas plurinominales al establecer que éstas no pueden contener más de dos candidatos del mismo sexo colocados de forma consecutiva en la ordenación de la lista (art. 2.3). Una disposición de esta legislación excluye la exigencia de cumplir las cuotas a los órganos con freguesias con un número igual o inferior a 750 electores y a los órganos de los municipios con 7.500 o un número menor de electores ${ }^{15}$. Hay que resaltar también que la norma en cuestión prevé una futura revisión de sus disposiciones, al disponer que, tras cinco años del inicio de su vigencia, la Asamblea de la República deberá evaluar su impacto en la promoción de la paridad entre hombres y mujeres y proceder a su revisión de acuerdo con esta evaluación ${ }^{16}$.

Las elecciones de 2009 indican que las cuotas tienen un impacto positivo en Portugal, pues el porcentaje de mujeres ha aumentado del $21,30 \%$ (en las elecciones de 2005) al $27,83 \%$. Sin embargo, como se ha observado que la eficacia de estas medidas puede variar en función del tiempo y de varios otros factores, habrá que esperar la evaluación de la Ley para que se verifique su impacto y los eventuales problemas relacionados con su aplicación y eficacia. Pese a ello, desde luego, es posible subrayar que el sistema electoral portugués de listas cerradas y bloqueadas y la previsión de alternancia entre mujeres y hombres en las listas se pueden considerar factores favorables para la eficacia de estas medida; por otro lado, la sanción limitada a la reducción del montante de subvenciones públicas para las campañas electorales constituye un elemento perjudicial a esta eficacia.

14 El art. 2.1 de esta Ley dispone que "se entiende por paridad, para los efectos de aplicación de la presente Ley, la representación mínima del 33,3\% de cada sexo en las listas”.

15 Art. 2.4 de la Ley Orgánica 3/2006.

16 Art. 8 de la Ley Orgánica 3/2006. 


\section{España}

En España, las primeras legislaciones que adoptaron cuotas electorales en función del sexo se introdujeron en el ámbito de las comunidades autónomas. En las Islas Baleares, se aprobó la Ley 6/2002, de 21 de junio, que estableció la siguiente redacción al artículo 16.4 de la Ley Electoral de la Comunidad Balear (Ley 8/1986, de 26 de noviembre):

Con la finalidad de hacer efectivo el principio de igualdad en la participación política, las candidaturas electorales deberán contener una presencia equilibrada de hombres y mujeres. Las listas se integrarán por candidatos de uno y otro sexo ordenados de forma alternativa.

Así pues, esta norma prevé el "sistema cremallera", es decir, la alternancia entre los sexos en las listas. La Comunidad Autónoma de Castilla-La Mancha adoptó una norma similar por medio de la aprobación de la Ley 11/2002, de 27 de junio, que añadió el apartado 1 bis al artículo 23 de la Ley electoral castellano-manchega ${ }^{17}$, estableciendo que

Para garantizar el principio de igualdad en la representación política, las candidaturas que presenten los partidos políticos, federaciones, coaliciones o agrupaciones de electores, alternarán hombres y mujeres, ocupando los de un sexo los puestos pares y los del otro los impares. La Junta Electoral sólo aceptará aquellas candidaturas que cumplan este precepto tanto para los candidatos como para los suplentes.

Aunque estas dos legislaciones se apliquen en la actualidad, han tenido anteriormente su vigencia suspensa como consecuencia de la interposición de recursos de inconstitucionalidad ${ }^{18}$. Éstos se

Ley $5 / 1986$, de 23 de diciembre.

18 Recursos de inconstitucionalidad n. 5536/2002 contra la Ley 6/2002, de 21 de junio, de las Islas Baleares, y n. 5537/2002, contra la Ley 11/2002, de 27 de junio, de Castilla-La Mancha. El 15 de octubre de 2002 en Tribunal Constitucional admitió a trámite los dos mencionados recursos de inconstitucionalidad y suspendió la vigencia y aplicación de los preceptos impugnados, de 
interpusieron por parte del presidente del Gobierno, en la época, representado por el Partido Popular, contrario a este tipo de medidas, como se ha mencionado anteriormente.

Tras el cambio de Ejecutivo en España, en 2004, mediante la victoria del Partido Socialista Obrero Español, se aprobaron otras dos leyes autonómicas que adoptaron cuotas electorales. El País Vasco aprobó la Ley 4/2005, de 18 de febrero, para la Igualdad de Mujeres y Hombres, que, entre un amplio conjunto de medidas, prevé la paridad en las candidaturas al Parlamento y a las Juntas Generales de los Territorios Históricos de Araba, Bizcaia y Gipuzkoa, al establecer que

las candidaturas que presenten los partidos políticos, federaciones, coaliciones o agrupaciones de personas electoras estarán integradas por al menos un $50 \%$ de mujeres. Se mantendrá esa proporción en el conjunto de la lista de candidatos y candidatas y en cada tramo de seis nombres. Las juntas electorales de zona competentes sólo admitirán aquellas candidaturas que cumplan lo señalado en este artículo, tanto para las personas candidatas como para las suplentes ${ }^{19}$.

Aunque esta Ley fue sometida a recurso de inconstitucionalidad por 62 diputados del Grupo Parlamentario Popular en el Congreso, tal recurso no lleva aparejada la suspensión de la aplicación de la norma impugnada. De ahí que las elecciones al Parlamento Vasco, celebradas en 2005, pudieran realizarse bajo la vigencia de esta normativa (MARTÍNEZ ALARCÓN, 2007).

conformidad con lo que establece el artículo 161.2 de la Constitución Española: "El Gobierno podrá impugnar ante el Tribunal Constitucional las disposiciones y resoluciones adoptadas por los órganos de las Comunidades Autónomas. La impugnación producirá la suspensión de la disposición o resolución recurrida, pero el Tribunal, en su caso, deberá ratificarla o levantarla en un plazo no superior a cinco meses". El Tribunal ratificó la suspensión de estas leyes autonómicas; en el caso de la ley balear, mediante el Auto de 14 de enero de 2003 (BOE n. 24, de 28 de enero de 2003), y en el caso de la ley castellano-manchega, mediante el Auto de 26 de febrero de 2003 (BOE n. 67, de 19 de marzo de 2003).

19 La Ley $4 / 2005$ prevé esta norma en las disposiciones finales cuarta y quinta, que modifican la Ley 5/1990, de 15 de junio (de elecciones al Parlamento Vasco) y la Ley 1/1987, de 27 de marzo (de Elecciones para las Juntas Generales de los Territorios Históricos de Araba, Bizkaia y Guipúzcoa), respectivamente. 
Andalucía también ha establecido la paridad en las candidaturas electorales por medio de la Ley 5/2005, de 8 de abril. Ésta modifica el artículo 23 de la Ley Electoral Andaluza, disponiendo que

La presentación de candidaturas en la que se alternarán hombres y mujeres habrá de realizarse entre el decimoquinto y el vigésimo días posteriores a la convocatoria, mediante listas que deben incluir tantos candidatos como escaños a elegir por cada circunscripción y, además, cuatro candidatos suplentes, expresándose el orden de colocación de todos ellos, ocupando los de un sexo los puestos impares y los del otro los pares.

En el marco nacional, entre los años 2001 y 2004, se presentaron varias propuestas de alteración de la Ley Orgánica del Régimen Electoral General (LOREG), con el fin de garantizar una presencia equilibrada de mujeres y hombres en las listas electorales. Plantearon tales propuestas el Grupo Parlamentario Socialista, el Grupo Parlamentario Federal de Izquierda Unida, el Grupo Parlamentario Mixto y el Grupo Parlamentario Izquierda Verde/Izquierda Unida/Iniciativa per Catalunya-Verds. No obstante, tales proposiciones de ley no tuvieron éxito en el Parlamento, principalmente por el desacuerdo del Grupo Popular.

Durante el nuevo gobierno socialista, éste presentó, en julio de 2006, un Proyecto de Ley Orgánica para la igualdad efectiva de mujeres y hombres, que fue aprobado el año siguiente y constituye la actual Ley Orgánica 3/2007, de 22 de marzo. Como se ha observado anteriormente, entre varias otras medidas, esta norma estableció cuotas electorales por razón de sexo del $40 \%$, por medio de una disposición que añade el artículo 44 bis a la Ley Orgánica del Régimen Electoral General (LOREG). El apartado uno de este artículo dispone que

las candidaturas que se presenten para las elecciones de diputados al Congreso, municipales y de miembros de los consejos insulares y de los cabildos insulares canarios en los términos previstos en esta Ley, diputados al Parlamento Europeo y miembros de las Asambleas Legislativas de las Comunidades Autónomas deberán tener una composición equilibrada de mujeres y hombres, de forma que en el 
conjunto de la lista los candidatos de cada uno de los sexos supongan como mínimo el cuarenta por ciento. Cuando el número de puestos a cubrir sea inferior a cinco, la proporción de mujeres y hombres será lo más cercana posible al equilibrio numérico.

Por otro lado, se establece la posibilidad de que las leyes autonómicas reguladoras de los regímenes electorales introduzcan medidas para favorecer una mayor presencia de mujeres en las candidaturas a las Asambleas Legislativas. Esta disposición legitima las mencionadas normas de las Comunidades Autónomas que han previsto un porcentaje mínimo de mujeres del 50\%, así como el "sistema cremallera" 20 . Como se dijo cuando se consideró la infrarrepresentación de la mujer en España, la Ley de Igualdad no obliga a la alternancia entre los sexos en las candidaturas, sino que impone la proporción mínima del $40 \%$ en cada tramo de cinco puestos, es decir, en cada grupo de cinco candidatos, se debe incluir al menos dos personas del mismo sexo (mujeres u hombres). Para el caso del Senado, se prevé que, cuando las candidaturas se agrupen en listas, estas deberán tener una composición de mujeres y hombres lo más cercana posible al equilibrio numérico.

La norma añade una disposición transitoria a la LOREG para las convocatorias a elecciones municipales que se produjeran antes de 2011. En este período, es decir, hasta el 2010, la cuota electoral no se exigió en los municipios con un número de habitantes igual o inferior a 5.000. Por otro lado, se prevé una norma de carácter permanente que exime de la exigencia de cumplir la cuota las candidaturas que se presenten en los municipios con un número de residentes igual o inferior a 3.000 y en las islas con un número de residentes igual o inferior a 5.000 .

En mayo de 2007, el Juzgado de lo Contencioso-Administrativo n. 1 de Santa Cruz de Tenerife planteó cuestión de constitucionalidad en relación con la introducción del artículo 44 bis a la LOREG por la Ley

En lo que respecta al sistema cremallera, hay que exceptuar el País Vasco, que no lo ha adoptado. 
de Igualdad. Tal cuestión se derivó de un recurso contencioso-electoral interpuesto por el Partido Popular, por el rechazo de una candidatura compuesta exclusivamente por mujeres presentada por dicha formación política a las elecciones municipales de Garachico. En junio de este mismo año, más de cincuenta diputados del Grupo Popular han provocado la interposición de un recurso de inconstitucionalidad contra la disposición adicional segunda da la citada Ley. El Tribunal Constitucional, por medio de la Sentencia 12/2008, de 29 de enero, ha decidido desestimar las dos demandas ${ }^{21}$.

En marzo de 2008, se han realizado las primeras elecciones generales durante la vigencia de las modificaciones a la LOREG introducidas por la Ley de Igualdad. Como se ha observado, el hecho de que los partidos políticos hubieran situado a las mujeres mayoritariamente en los puestos de difícil salida ha impedido que la norma produjera una mayor eficacia a fin de aumentar el porcentaje de participación de mujeres en el Parlamento. Es oportuno subrayar también que la proporción de mujeres en el Congreso antes de las cuotas ya era próxima al 40\%, por lo que no hay como verificar un impacto tan significativo, a no ser que el porcentaje sea ampliado.

21 En sentencias anteriores, el Tribunal Constitucional ha tenido la oportunidad de pronunciarse sobre algunos problemas prácticos relacionados con la aplicación del art. 44 bis de la LOREG. Se tratan de decisiones proferidas en razón de la interposición de recursos de amparo por parte de partidos políticos y agrupaciones de electores que incumplieron, por error, el porcentaje mínimo de candidaturas para cada sexo. En estos casos, el Tribunal Constitucional ordenó que se retrotrajeran las actuaciones de la Junta Electoral al momento anterior a la proclamación de candidatos, de forma que los partidos y agrupaciones pudieran subsanar los defectos señalados. No obstante, como en el caso de Garachico, en otra ocasión, la lista presentada incumplía de forma explícita la legislación electoral. Se trata de la candidatura presentada por Falange Española, de las JONS, en la localidad de Brunete, que incluía diez mujeres y tres varones. En este asunto, el Tribunal Constitucional también ordenó retrotraer las actuaciones de la Junta Electoral para posibilitar la subsanación de los defectos apreciados (STC 108/2007, de 10 de mayo). Sin embargo, alegando imposibilidad material para corregir la candidatura, el partido agotó la vía contenciosa e interpuso otro recurso de amparo ante el Tribunal Constitucional. En la Sentencia 127/2007, de 22 de mayo, este órgano deniega el amparo por entender que resulta posible cumplir la exigencia de la ley, y considerar que lo que pretende el partido es únicamente cuestionar la legitimidad de los preceptos del art. 44 bis de la LOREG (Sobre eso, véase BIGLINO CAMPOS, 2008, p. 167-169). 
No obstante, es necesario analizar los datos para que no se haga un juicio incorrecto. El Partido Socialista ha elegido, en el año 2008, 73 diputadas, es decir, un $43,20 \%$ del total de los candidatos socialistas elegidos (169) eran mujeres, lo que refleja un razonable equilibrio entre los sexos. No obstante, hay que reconocer que este partido ha elegido tres diputadas, menos que en la legislatura anterior. Por el Partido Popular, han sido elegidas, en el año 2008, 46 diputadas, lo que representa un $29,87 \%$ del total de los elegidos por este agrupamiento político (154). En el caso del Partido Popular, han sido elegidas cuatro mujeres más que en 2004, cuando este grupo político ha logrado obtener 42 escaños para diputadas. Convergència i Unió (CIU), que en la legislatura de 2004 sólo había elegido una mujer de un total de 10 personas elegidas, eligió, en el año 2008, cuatro mujeres, también de un conjunto de 10 elegidos, lo que forma un $40 \%$ de mujeres y representa un significativo aumento.

Para el Partido Nacionalista Vasco (PNV), la pérdida de un escaño en las elecciones de 2008 (en las que han elegido 6 diputadas) representó también la pérdida de la única diputada que tenía. Izquierda Unida, a su vez, ha perdido tres escaños en 2008 (se han elegido dos diputados), así como a las dos únicas diputadas que tenía. Cada uno de los demás partidos eligieron una mujer, lo que representa al menos el $50 \%$ del total de elegidos por partido, que constituye uno o dos candidatos, de acuerdo con el caso ${ }^{22}$ (lo que no supone una baja en relación con las elecciones de 2004).

Así, pues, los partidos que obtuvieron menos escaños para las mujeres en 2008, en relación con el 2004, fueron el PSOE, PNV e IU. Por otro lado, los partidos que en 2008 no lograron elegir al menos a un $40 \%$ de mujeres fueron el PP, PNV y IU. Para aumentar el total de mujeres en el Congreso de los Diputados, es necesario que estos tres partidos, principalmente, pasen a ampliar el número de candidatas en los puestos de salida. Se puede constatar, por lo tanto, la importancia

22 El Bloque Nacionalista Gallego (BNG) y Coalición Canaria (CC) han elegido 2 diputados cada uno. Unión Progreso y Democracia (UPyD) y Nafarroa Bai (Na-Bai) han elegido 1 diputada cada uno. 
de la adopción del "sistema cremallera" para el incremento de la cifra de diputadas.

Hay que resaltar que no es exacta la afirmación divulgada por algunos medios de comunicación de que la Ley de Igualdad ha "restado" una mujer del Congreso en el año 2008. Por el contrario, este año, han sido elegidas 127 candidatas, una más que en 2004, cuando han resultado elegidas 126 candidatas $(36 \%)^{23}$. Sin embargo, tras las elecciones, tanto mujeres como hombres pueden desistir de acceder al puesto o causar baja, por diversos motivos, lo que posibilita la alteración de la composición de la Cámara en relación con el sexo. En el presente año, por ejemplo, algunas mujeres que han causado baja después de su elección han sido sustituidas por hombres (la afirmación contraria también es verdadera, pero en menor medida), lo que conlleva que, en la actualidad, sólo 124 diputadas ocupen un escaño en la Cámara Baja $(35,42 \%)$.

Por otro lado, es oportuno observar que, en el ámbito municipal, el número de mujeres elegidas ha aumentado de forma significativa tras la aplicación de las cuotas, ya que, en las elecciones municipales de 2003 , este porcentaje fue de $32,20 \%$. En las elecciones de 2007 , las primeras realizadas después de la Ley de Igualdad, este porcentaje se incrementó al 39,40\%. No obstante, también en este ámbito se observa que las mujeres ocupan mayoritariamente las peores posiciones de las listas. Los datos de investigaciones realizadas demuestran que, en las elecciones de 2007 , las candidatas femeninas sólo ocuparon la primera posición en el $20 \%$ de las listas electorales presentadas (VENTURA FRANCH, 2009). Sin embargo, en la segunda y tercera posición, el porcentaje aumenta para una media del $40 \%$, y en la cuarta y quinta, representan el $50 \%$ o más. Así, se verifica que, a pesar del evidente "techo de cristal" localizado en la primera posición de la lista, en las

23 Se afirma que, en 2004, se sentaron en los escaños del hemiciclo 127 diputadas. No obstante, esto no significa que hubieran sido electas 127 parlamentarias. Los datos presentados en este trabajo (127 mujeres electas en 2007 y 126 en 2004) fueron fornecidos por el propio Congreso Nacional y confirman los porcentajes divulgados por el Instituto de la Mujer. 
demás posiciones la proporción de las mujeres tiende al equilibrio, lo que contribuye para una mayor eficacia de las cuotas.

En lo que concierne a los parlamentos autonómicos, también se ha observado un aumento de mujeres elegidas después de la Ley de Igualdad de 2007. En el año 2006, el porcentaje medio de representantes femeninas en los parlamentos autonómicos españoles era del 37,77\%, y, en 2007, creció al $41,11 \%{ }^{24}$. Es importante observar que las Comunidades Autónomas que han aprobado leyes estableciendo la alternancia entre mujeres y hombres en las listas electorales son algunas de las que presentan el mayor número de mujeres en sus parlamentos: Castilla-LaMancha $(53,19 \%)$, Baleares $(49,15 \%)$ y Andalucía $(45,87 \%)^{25}$.

\section{Conclusión}

Bélgica fue el primer país en adoptar las cuotas electorales legales, lo que hizo en 1994, sin reforma constitucional previa y sin que fuera discutida su constitucionalidad ante la Corte de Arbitraje. No obstante, posteriormente, se reformó la Constitución y se aprobó una nueva ley. En Francia e Italia, en la década de 1990, algunas iniciativas de adopción de cuotas electorales llegaron a convertirse en ley. No obstante, tales iniciativas resultaron frustradas en razón de la declaración de inconstitucionalidad por parte de los Tribunales Constitucionales respectivos. De esta forma, estos países reformaron su Constitución para favorecer la cobertura constitucional de las cuotas electorales legales. De hecho, después de tales reformas, en estos países, se han aprobado leyes con previsión de cuotas que han sido consideradas legítimas por parte de sus Tribunales Constitucionales. La reciente ley de paridad portuguesa (Ley Orgánica 3/2006) también ha sido precedida de una reforma constitucional. En España, la ley que estableció cuotas electorales (Ley Orgánica 3/2007) fue considerada legítima por parte del Tribunal Constitucional sin que se hubiera realizado

\footnotetext{
24 INSTITUTO DE LA MUJER. http://www.inmujer.es.

25 Ídem.
} 
reforma constitucional alguna. Además, entre los países estudiados, España es el único que no ha modificado su Constitución para insertar una disposición específica relativa a la igualdad entre mujeres y hombres en el acceso al poder político.

Sobre la eficacia de estas medidas en los países europeos estudiados, se observan situaciones diversas. En Bélgica, los números indican que estas medidas tuvieron éxito y contribuyeron a la ampliación de la proporción de mujeres parlamentarias. En Francia, se ha observado un impacto positivo en las elecciones municipales y para los Consejos Regionales. Sin embargo, en las elecciones a las Asambleas Departamentales, al Senado y a la Asamblea Nacional, los números de mujeres elegidas permanecieron bajos. En Portugal, las elecciones de 2009 indican que las cuotas tienen un impacto positivo. Como se ha observado, la eficacia de estas medidas puede variar en función del tiempo y de otros varios factores, razón por la cual habrá que esperar a la evaluación de la Ley para que se pueda verificar su impacto y los eventuales problemas relacionados con su aplicación y eficacia.

En España, no se ha constatado una diferencia en el número de mujeres elegidas al Congreso después de la aplicación de las cuotas. El hecho de que los partidos políticos hubieran situado a las mujeres mayoritariamente en los puestos de difícil salida ha impedido que la norma produjera una mayor eficacia para aumentar el porcentaje de participación de mujeres en el parlamento. Es oportuno subrayar también que la proporción de mujeres en el Congreso antes de las cuotas ya era próxima al $40 \%$, por lo que no hay manera de verificar un impacto significativo, a no ser que el porcentaje resulte ampliado. Por otro lado, se ha observado que, en el ámbito municipal, el número de mujeres elegidas ha aumentado de forma significativa tras la aplicación de las cuotas. En lo que concierne a los parlamentos autonómicos, también se ha constatado un aumento de mujeres elegidas después de la Ley de Igualdad de 2007. Es importante subrayar que las Comunidades Autónomas que han aprobado leyes estableciendo la alternancia entre mujeres y hombres en las listas electorales son algunas de las que tienen un mayor número de mujeres en sus parlamentos. 


\section{Referencias}

AMARAL, Maria Lúcia. Las mujeres en el derecho constitucional: el caso portugués. In: MUJER y Constitución en España. Madrid: Centro de Estudios Políticos y Constitucionales, 2000. p. 155-174.

BIGLINO CAMPOS, Paloma. La composición equilibrada de las candidaturas electorales: primeras experiencias. In: ENTRE la ética, la política y el derecho: estudios en homenaje al profesor Gregorio Peces-Barba. Madrid: Dykinson, 2008. v. 1, p. 167-169.

CECCHERINI, Eleonora. La igualdad de los sexos en la representación política: la experiencia italiana. Revista de Derecho Constitucional Europeo, Granada, n. 6, p. 325-353, 2006.

JANSON, Jane; VALIENTE FERNÁNDEZ, Celia. El movimiento a favor de la democracia paritaria en Francia y España. Revista Española de Ciencia Política, Madrid, n. 5, p. 79-110, oct. 2001.

LENOIR, Noëlle. The representation of women in politics: from quotas to parity in elections. International and Comparative Law Quarterly, Cambridge, v. 50, n. 2, p. 217-247, Apr. 2001.

MATEO DÍAZ, Mercedes. Les quotas sont-ils utiles? L'éfficacité imparfaite des mesures de discrimination positive dans les lois électorales belges. Revue Française de Science Politique, Paris, v. 53, n. 5, p. 791-815, 2003.

MARTÍNEZ ALARCÓN. Cuota electoral de mujeres y derecho constitucional. Madrid: Congreso de los Diputados, 2007.

MATEO DÍAZ, Mercedes. Les quotas sont-ils utiles? L'éfficacité imparfaite des mesures de discrimination positive dans les lois électorales belges. Revue Française de Science Politique, v. 53, n. 5, p. 791-815, 2003.

MEIER, Petra. Bélgica: una buena práctica en perspectiva. In: DAHLERUP, Drude; FREIDENVALL, Lenita. Sistemas electorales de cuotas de género y su aplicación en Europa. Bruselas: Parlamento Europeo, 2008. p. 45-54. 
. Experiencias de reformas electorales en algunos países de la Unión Europea: iniciativas en Bélgica y Portugal. In: SAAVEDRA RUIZ, Paloma (Org.). Hacia una democracia paritaria: análisis y revisión de las leyes electorales vigentes. Madrid: Coordinadora Española para el Lobby Europeo de Mujeres, 1999. p. 201-223.

SINEAU, Mariette. Francia: Parité ante la ley. In: DAHLERUP, Drude; FREIDENVALL, Lenita. Sistemas electorales de cuotas de género y su aplicación en Europa. Bruselas: Parlamento Europeo, 2008. p. 5564.

VENTURA FRANCH, Asunción; ROMANÍ SANCHO, Lucía. Ley de igualdad y elecciones municipales: un análisis de la provincia de Castellón. Corts: Anuario de Derecho Parlamentario, Valencia, n. 21, 2009, p. 187-211.

Recebido em: 11/11/12

Aprovado em: 28/12/12 